\title{
Microstructural Evolution and Phase Transformation of a Ti-5Nb-5Al Alloy During Annealing Treatment
}

\author{
Yu Dai ${ }^{a}$ Min Song ${ }^{b *(1)}$ \\ ${ }^{a}$ School of Materials Science and Engineering, Nanchang University, Nanchang, 330031, P.R. China
${ }^{b}$ State Key Laboratory of Powder Metallurgy, Central South University, Changsha 410083, P.R. China
}

Received: September 06, 2019; Accepted: October 02, 2019

\begin{abstract}
The microstructural evolution and phase transformation of a Ti-5Nb-5Al alloy during isothermal annealing treatment were studied in this paper. The microstructural evolution was analyzed by X-ray diffraction (XRD), scanning electron microscope (SEM) equipped with X-ray energy dispersive spectroscopy (EDS) and electron backscattering diffraction (EBSD). The first-principle calculation by density functional theory method was performed to analyze the effect of niobium diffusion on the $\alpha$ to $\beta$ transformation. The results showed that the $\alpha$ phase initially grew by merging neighboring grains and then by boundary splitting with increasing the solution time below the $\beta$-transus temperature. However, the $\alpha$ phase disappeared absolutely above the $\beta$-transus temperature. According to the thermodynamic analysis by DFT calculations, the diffusion of niobium from $\beta$ to $\alpha$ phase can promote the $\alpha$ to $\beta$ transformation. After quenching in water, the acicular $\alpha$ ' phase precipitated from $\beta$ matrix with an orientation relationship of $\{110\}_{\text {bcc }} \|\{0001\}_{\text {hcp }}$ and $<111>_{\text {bcc }} \|<11 \overline{2} 0>_{\text {hcp }}$, and thus increased the hardness of the alloy.
\end{abstract}

Keywords: Titanium alloy, Microstructural evolution, Hardness, Diffusion, First-principle calculation.

\section{Introduction}

Over the past decades, titanium alloys have been widely used in various fields, such as aerospace, biomedical and chemical industries, due to their high strength, low Young's modulus, good biocompatibility and excellent corrosion resistance $^{1-4 .}$ In general, mechanical properties of materials depend significantly on their microstructures. Thus, previous investigations ${ }^{5-7}$ have been focusing on the microstructural evolution of titanium alloys in order to improve the mechanical properties. ZHANG et al. ${ }^{5}$ studied plastic flow behavior and microstructural evolution of a Ti-6Al-4V alloy with different initial microstructures during subtransus hot deformation, from which a homogenous ultrafine-grained structure was developed from a fully martensitic initial microstructure via hot deformation. HUANG et al. ${ }^{6}$ revealed the effects of microstructures on the strength and plasticity of a metastable $\beta$ - $\beta$ Ti-5Al-5Mo-5V-3Cr-1Zr alloy (Ti-55531). They found that the existence of twinning structure can greatly enhance the ductility of lamellar microstructure during plastic deformation. SHI et al. ${ }^{7}$ also investigated microstructural evolution and mechanical properties of TC21 titanium alloy under different heat treatment conditions and found that the existence of crisscross $\alpha$ plates with large thickness contributed to the improvement of the fracture toughness.

In general, $\alpha$ and $\beta$ phases are the most common phases in titanium alloys. The $\alpha$ phase presents as a hexagonal close-packed (HCP) structure, which is stable at room temperature. Generally, the $\alpha$ phase has three different microstructural morphologies, namely the lamellar, equiaxed and basket-weave shapes ${ }^{8,9}$.

*e-mail: msong@csu.edu.cn
The $\beta$ phase exhibits a body-centered cubic (BCC) structure, which is stable at high temperature. The $\beta$ phase always exists in the form of equiaxed microstructure at high temperature ${ }^{8}$. At a specific transition temperature, $\alpha$ and $\beta$ phases can transform between each other. For example, the $\alpha$ phase transforms to the $\beta$ phase above $882{ }^{\circ} \mathrm{C}$ in pure titanium ${ }^{10}$ and above $860^{\circ} \mathrm{C}$ in Ti-5Al-5Mo-5V-1Fe-1Cr alloy ${ }^{11}$. There are also other metastable phases in titanium alloy, such as $\omega$ phase (simple hexagonal structure), $\alpha$ " phase (HCP structure) and $\alpha$ " phase (monoclinic structure). Typically, isothermal $\omega\left(\omega_{\text {iso }}\right)$ phase forms uniformly in the $\beta$ matrix at a lower thermal treatment temperature compared to the $\beta$-to- $\alpha$ transition temperature, which contributes to the homogeneous nucleation of the $\alpha$ precipitates with a fine-scaled size $e^{9,12}$. The formation of $\alpha^{\prime}$ and $\alpha$ " phases in the $\beta$ matrix of titanium alloys was observed during rapid cooling from high temperature, as well as during stress-induced transformation in previous studies ${ }^{13,14}$.

As mentioned-above, the changes in the microstructure and phase transformation have significant impacts on the mechanical properties of titanium alloys. Meanwhile, the diffusion of alloying elements also affects the phase transformation during heat treatment. Previously, MISHIN and HERZIG systematically summarized the Al diffusion in different phases in Ti-Al system ${ }^{15}$. However, the effect of $\mathrm{Nb}$ diffusion on the phase transition of Ti-Al system has rarely been studied. It should be noted that $\mathrm{Nb}$ element can stabilize $\beta$ phase ${ }^{16}$. Thus, in this paper, a Ti-5Nb-5Al alloy was heat-treated at different solution temperatures for varied time, and the influence of $\mathrm{Nb}$ element diffusion on the phase transformation was studied by both experiments and density functional theory (DFT) calculations. 


\section{Materials and Methods}

\subsection{Materials and experimental method}

The initial material used in this work consists of $90 \mathrm{wt} . \%$ Ti, 5 wt. $\% \mathrm{Nb}$ and 5 wt. \% Al. Samples with a diameter of $8 \mathrm{~mm}$ and a height of $5 \mathrm{~mm}$ were cut from the initial material, and then annealed for 30 minutes at $950{ }^{\circ} \mathrm{C}, 970^{\circ} \mathrm{C}, 980^{\circ} \mathrm{C}$ and $1000^{\circ} \mathrm{C}$, respectively, followed by water cooling to the room temperature (as shown in Table 1). These specimens were named 950-30, 970-30, 980-30 and 1000-30, respectively. In order to better explain the microstructural evolution at different annealing periods, another group of samples were annealed for 15 minutes at $950{ }^{\circ} \mathrm{C}, 970{ }^{\circ} \mathrm{C}$ and $1000{ }^{\circ} \mathrm{C}$, respectively, and defined as 950-15, 970-15 and 1000-15, respectively. The hardness of the specimens was obtained by using the Vickers microhardness tester, with a load of $2 \mathrm{~kg}$ for $15 \mathrm{~s}$. At least 5 independent hardness tests were performed on each sample. The phase constitution was analyzed by $\mathrm{X}$-ray diffraction (XRD) using $\mathrm{Cu} \mathrm{K} \alpha$ radiation $(40 \mathrm{kV}, 200 \mathrm{~mA})$. X-ray patterns were collected in the range $2 \theta=10^{\circ} \sim 90^{\circ}$ with the step size of $0.02^{\circ}$. An FEI Helios NanoLab G3 UC scanning electron microscope (SEM) equipped with X-ray energy dispersive spectroscopy (EDS) and electron backscattering diffraction (EBSD) was applied to characterize the microstructure of the samples and identify the phase compositions. SEM specimens were initially ground, mechanically polished, and then chemically etched using a solution of hydrofluoric acid (4 vol. \%), nitric acid (6 vol. $\%)$ and water (90 vol. \%). Specimens for EBSD analysis were prepared as follows. Firstly, discs with a diameter of 3 $\mathrm{mm}$ were cut from the annealed samples. Secondly, the discs were mechanically ground to $\sim 80 \mathrm{~m}$ in thickness. Thirdly, the ground discs were electropolished using a solution of 5 $\%$ perchloric acid, $35 \%$ butanol and $60 \%$ methanol at -30 ${ }^{\circ} \mathrm{C}$ with an applied voltage of $30 \mathrm{~V}$.

Table 1. The heat treatment conditions of Ti-5Nb-5Al alloy.

\begin{tabular}{lccc}
\hline Sample & $\begin{array}{c}\text { Temperature } \\
\left({ }^{\circ} \mathbf{C}\right)\end{array}$ & $\begin{array}{c}\text { Time } \\
(\mathbf{m i n u t e s})\end{array}$ & Cooling mode \\
\hline Original sample & -- & -- & -- \\
$950-15$ & 950 & 15 & Water cooling \\
$950-30$ & 950 & 30 & Water cooling \\
$970-15$ & 970 & 15 & Water cooling \\
$970-30$ & 970 & 30 & Water cooling \\
$980-30$ & 980 & 30 & Water cooling \\
$1000-15$ & 1000 & 15 & Water cooling \\
$1000-30$ & 1000 & 30 & Water cooling \\
\hline
\end{tabular}

\subsection{Calculation method}

In order to better understand the impact of $\mathrm{Nb}$ element on the $\alpha$-to- $\beta$ phase transformation in the alloy, the DFT method was used to analyze the thermodynamic stability of the $\alpha$ and $\beta$ phases with increasing the $\mathrm{Nb}$ content in titanium.
The first-principle calculations by DFT method were carried out using the Vienna $\mathrm{Ab}$ initio Simulation Package (VASP) code ${ }^{17,18}$. In our calculations, generalized gradient approximation (GGA) was used for the exchange correlation function with the Perdew-Becke-Erzenh of (PBE) parameterization ${ }^{19}$. The interaction between valence electrons and ionic cores was described using PAW pseudopotentials ${ }^{20}$. The number of valence electrons in the pseudopotentials are $10\left(3 \mathrm{~d}^{8}, 4 \mathrm{~s}^{2}\right)$, and $11\left(4 \mathrm{p}^{8}, 5 \mathrm{~s}^{1}, 4 \mathrm{~d}^{2}\right)$ for $\mathrm{Ti}$ and $\mathrm{Nb}$, respectively. The plane-wave cutoff energy was chosen to be $400 \mathrm{eV}$ and a $8 \times 8 \times 6$ Monkhorst-Pack mesh was used to sample the Brillouin zone. The supercells of $\mathrm{Ti}_{\mathrm{x}} \mathrm{Nb}_{1-\mathrm{x}}$ alloy consist of $2 \times 2 \times 3$ elementary hexagonal or body-centered cubic unit cells with a total of 24 atoms. The fraction of $\mathrm{Nb}$ in the alloys varied from 4.17 at. $\%$ to 20.83 at. \%. Different local arrangements of atoms resulted in little change to the energy of systems with the same chemical composition and thus were not taken into consideration ${ }^{10}$. For structure relaxation, the supercells were fully relaxed with respect to both the volume and shape, as well as to all the atomic positions. Meanwhile, the supercells were optimized until the force on each atom is smaller than $0.01 \mathrm{eV} / \AA$.

\section{Results}

\subsection{Hardness}

Figure 1 shows the average hardness of the specimens after annealing for 30 minutes at different temperatures. It can be seen that the original sample without heat treatment has the lowest hardness value of $283 \mathrm{HV}$ and the 1000-30 sample has the highest hardness value of $328 \mathrm{HV}$. With increasing the solution temperature, the hardness of the alloy increases notably. However, there is a temporary slight decrease in hardness for sample 980-30, compared to the hardness of sample 970-30.

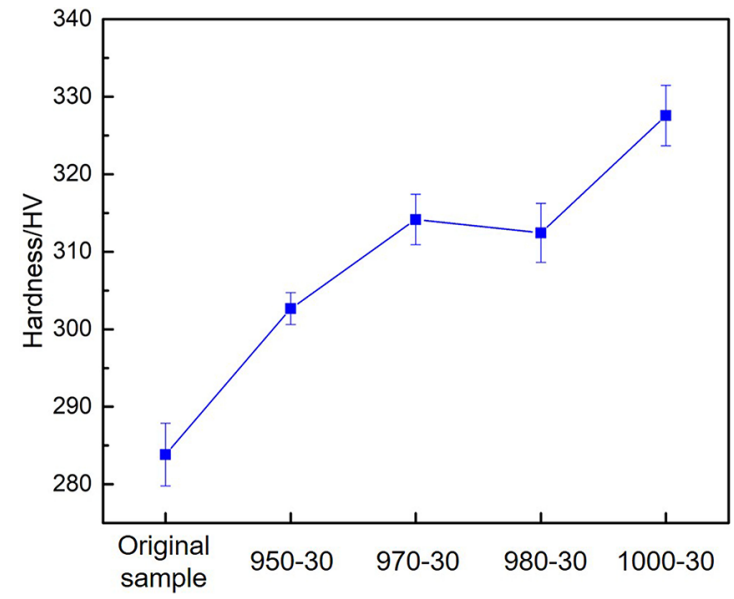

Figure 1. The harness of initial and annealed $\mathrm{Ti}-5 \mathrm{Nb}-5 \mathrm{Al}$ alloy at different temperatures. 


\section{$3.2 X R D$ results}

Figure 2 shows the XRD patterns of the samples after heat treatment. It can be seen that all the diffraction peaks are basically consistent with the standard peaks of HCP titanium. Furthermore, the strongest peaks at $2 \theta \approx 38.5^{\circ}$ in all samples are basically consistent with the standard peaks of BCC titanium. Meanwhile, the peaks at $2 \theta \approx 70^{\circ}$ in $970-15$ and $1000-30$ samples and the peaks at $2 \theta \approx 82^{\circ}$ in $970-15,970$ 30 and 980-30 samples are also basically consistent with the standard peaks of BCC titanium. Owing to lattice distortion resulted from the alloying elements and thermal dilation, all the peak positions in the XRD patterns are slightly deviated from the standard peak positions. In addition, no other phases were found in the XRD patterns. Therefore, all specimens consist of both the HCP $(\alpha)$ and BCC ( $\beta)$ phases.

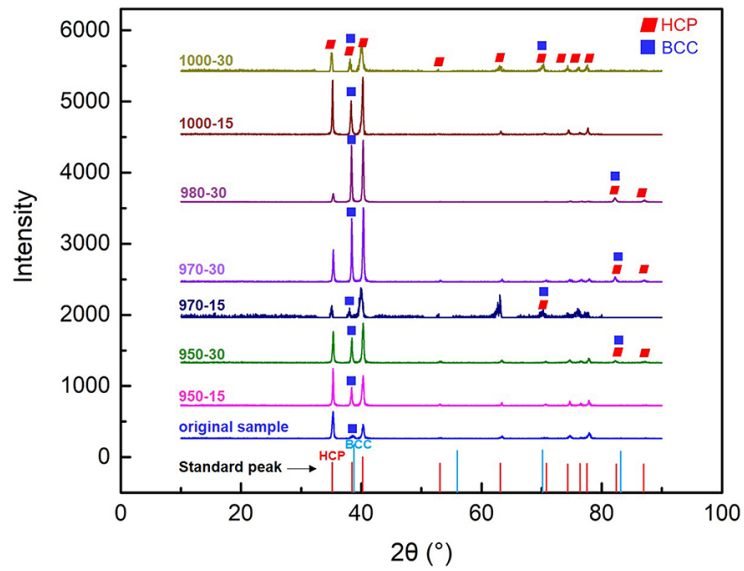

Figure 2. XRD patterns of initial and annealed Ti-5Nb-5Al alloy at different temperatures.

\subsection{Microstructures}

Figure 3 shows the SEM micrographs (secondary electrons) of the original sample. It can be seen from Figure 3 that the microstructure of the original sample contains both the $\beta$ and $\alpha$ phases. The average size of phase is about $1 \mu \mathrm{m}$. which was obtained by calculating the average value of 20 different grains of phase in SEM images. The grain size of phase in SEM images is measured by the 2D image analysis software of Image-Pro Plus 6.0. The size of other phases also used the same measuring method. The EDS analysis indicates a high concentration of $\mathrm{Nb}$ element in $\beta$ phase of the original sample, which reaches to $11.79 \mathrm{wt}$ \%, as shown in Table 2 .

Table 2. The EDS results of $\beta$ phase with different annealing temperatures and time.

\begin{tabular}{lcc}
\hline \multirow{2}{*}{ Heat treatment condition } & \multicolumn{2}{c}{ Elements } \\
\cline { 2 - 3 } & Al (wt. \%) & Nb (wt. \%) \\
\hline Original sample & 4.53 & 11.79 \\
$950-30$ & 4.68 & 6.43 \\
$970-30$ & 4.75 & 5.71 \\
$980-30$ & 4.91 & 5.42 \\
$1000-30$ & 4.66 & 5.22 \\
\hline
\end{tabular}

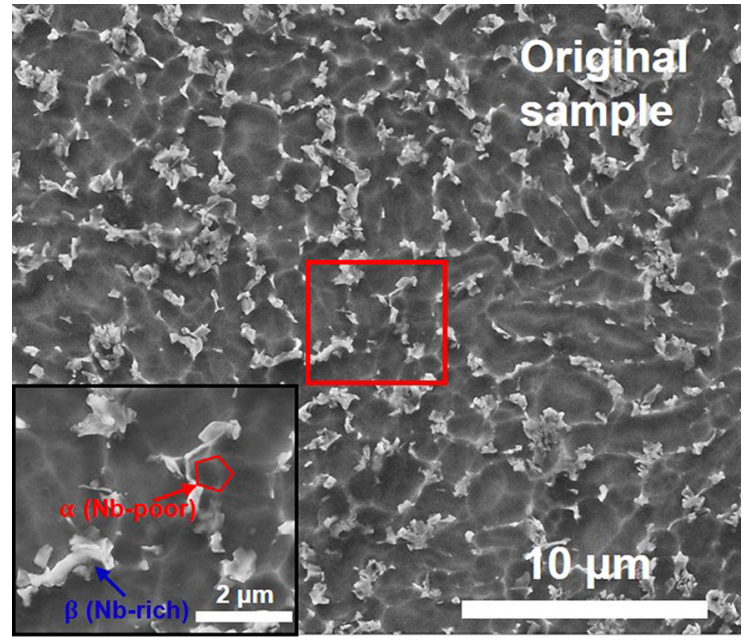

Figure 3. SEM image of the original sample.

Figure 4(a) to 4(f) shows the SEM micrographs of 950-15, 950-30, 970-30, 980-30, 1000-15 and 1000-30 samples, respectively. It can be seen that both the $\beta$ phase and equiaxial $\alpha$ phase coexist in 950-15, 950-30 and 97030 samples, as shown in Figure 4a, $4 b$ and 4c, respectively. Compared to the original sample, the grain sizes of $\alpha$ and $\beta$ phases in the $950-15$ sample are larger. Moreover, by increasing the annealing time from $15 \mathrm{~min}$ to $30 \mathrm{~min}$, the grain size of $\beta$ phase increases and the amount of $\alpha$ phase decreases (see 950-30 sample in Figure 4b). With increasing the annealing temperature to $970{ }^{\circ} \mathrm{C}$, the $\beta$ phase continues to grow and the amount of $\alpha$ phase decreases continuously (see 970-30 sample in Figure 4c), compared to 950-15 and 950-30 samples. Note that the grain size of $\beta$ phase is $2 \sim 15$ $\mu \mathrm{m}$ in 950-15 sample, 6 20 $\mu \mathrm{m}$ in 950-30 sample and 8 30 $\mu \mathrm{m}$ in $970-30$ sample, respectively. The $\beta$ phase grows at the cost of the elimination of $\alpha$ phase. The EDS analysis of the $\mathrm{Nb}$ concentration in $\alpha$ and $\beta$ phases in $950-15,950-30$ and 970-30 samples shows that $\mathrm{Nb}$ prefers to be enriched in $\beta$ phase but poor in $\alpha$ phase. The similar results were also observed in the original sample. However, compared to the original sample, $\mathrm{Nb}$ concentration in $\beta$ phase is reduced with increasing the annealing temperature. The reason may be that the concentration gradient of $\mathrm{Nb}$ in $\alpha$ and $\beta$ phases leads to the diffusion of $\mathrm{Nb}$ element from the $\beta$ phase (or $\mathrm{Nb}$-rich area) to the $\alpha$ phase (or Nb-poor area), which affects the relative stabilities of the two phases and thus promotes the $\alpha$-to- $\beta$ phase transformation. The effects of $\mathrm{Nb}$ content on the thermodynamic stability of $\alpha$ and $\beta$ phases will be discussed later in Section 4.2 by first-principle calculations. It is worth noting that the acicular structure can be observed in $\beta$ matrix in the 970-30 sample, as shown by the inserted figure in Figure 4c. Those acicular structures orienting in different directions intersect with each other with a crossing angle of $90^{\circ}$. According to the EDS analysis, it is confirmed that the acicular structure is composed of the martensite $\alpha^{\prime}$ phase, although that the chemical compositions between the acicular structures and $\beta$ matrix are similar (see in Table 3). 

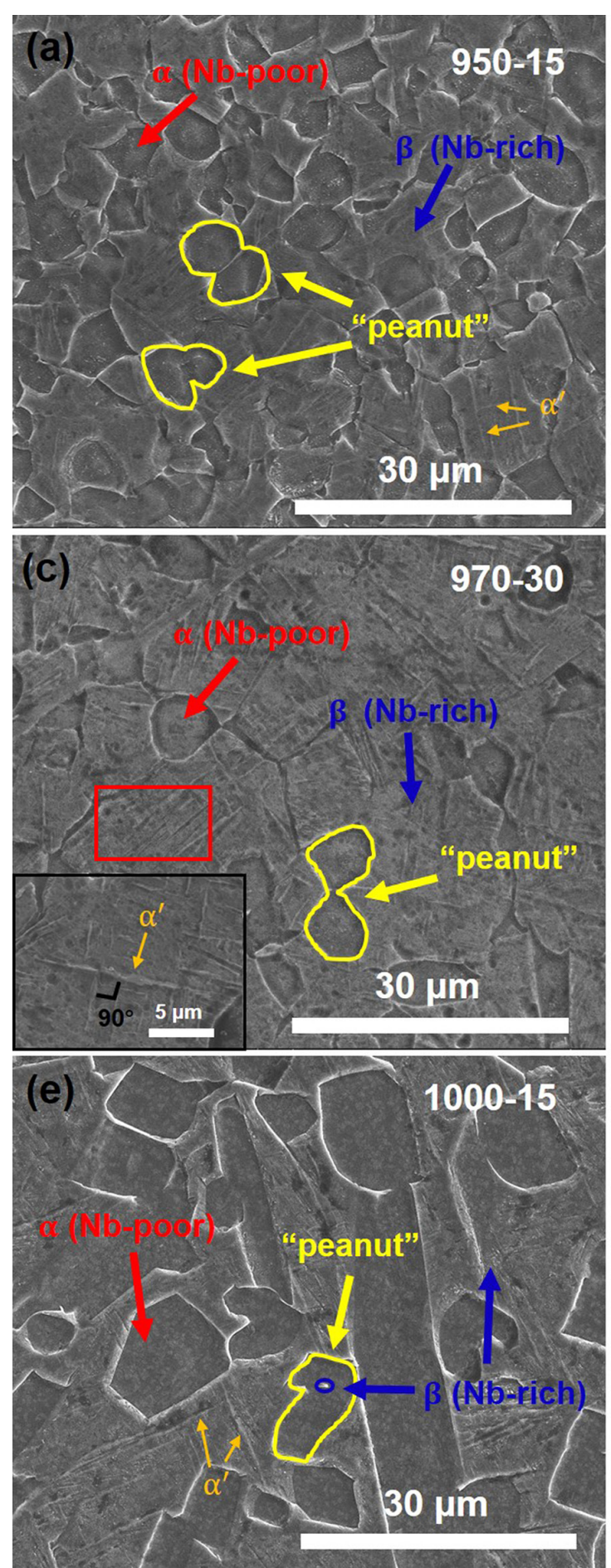

Figure 4. SEM images of (a) 950-15 sample, (b) 950-30 sample, (c) 970-30 sample, (d) 980-30 sample, (e) 1000-15 sample and (f) 1000-30 sample.

Table 3. The EDS results of $\alpha^{\prime}$ phase with different annealing temperatures and time.

\begin{tabular}{lcc}
\hline \multirow{2}{*}{ Heat treatment condition } & \multicolumn{2}{c}{ Elements } \\
\cline { 2 - 3 } & Al (wt. \%) & Nb (wt. \%) \\
\hline $980-30$ & 5.50 & 5.48 \\
$1000-30$ & 4.97 & 5.32 \\
\hline
\end{tabular}
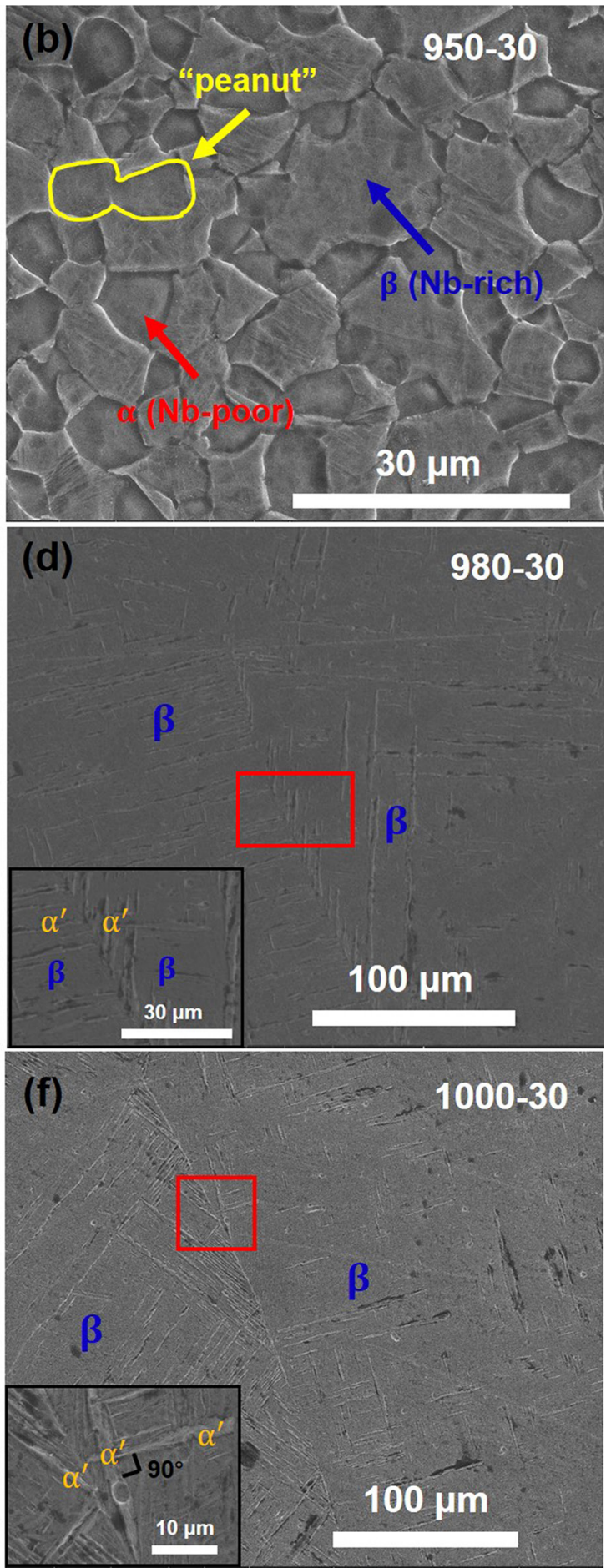

The microstructure in Figure 4e shows that 1000-15 sample consists of $\beta$ phase, $\alpha$ phase and acicular $\alpha$ ' phases. In addition, parts of the $\alpha$ grains are contiguous with a "peanut" shape, as is observed in Figure 4a to $4 \mathrm{c}$. The size of grains in the 1000-15 sample is the largest among all specimens and reaches a value of about $15 \mu \mathrm{m}$. Figure $4 \mathrm{f}$ shows the microstructure of 1000-30 sample. 
It can be seen from Figure $4 \mathrm{f}$ that the microstructure contains $\beta$ and acicular $\alpha^{\prime}$ phases, but without $\alpha$ phase. This indicates that the annealing temperature of $1000{ }^{\circ} \mathrm{C}$ is above the $\beta$-transus temperature. Therefore, annealing for enough time at $1000{ }^{\circ} \mathrm{C}$ results in the complete transformation of $\alpha$ phase to $\beta$ phase. The $\beta$ grain size in 1000-30 sample is about $600 \mu \mathrm{m}$, which is much larger than that in 950-30 and 970-30 samples. In addition, the acicular $\alpha^{\prime}$ phase grows significantly to a length of $10 \sim 160 \mu \mathrm{m}$ and a width less than $2 \mu \mathrm{m}$, and distributes mainly along the $\beta$ grain boundary. It should be noted that the same results were obtained in the 980-30 sample as those in 1000-30 sample, as seen in Figure 4d. It can thus be concluded that the $\beta$-transus temperature for this alloy lies between $970^{\circ} \mathrm{C}$ and $980^{\circ} \mathrm{C}$. In order to further verify the $\alpha$-to- $\beta$ phase transformation, the curves of the transformed fraction of $\alpha$ and $\beta$ phases via annealing time are shown in Figure 5. The fraction of $\beta$ and $\alpha$ phases were obtained by calculating the ratio of the total area of the $\beta$ or $\alpha$ phase in SEM images to the total area of the SEM image. The total area of the $\beta$ or $\alpha$ phase in SEM images is approximately measured by the $2 \mathrm{D}$ image analysis software of Image-Pro Plus 6.0. It is found that the fraction of $\beta$ phase increases and the fraction of $\alpha$ phase decreases with increasing the annealing time, as shown in Figure 5. It thus indicates that the $\alpha$-to- $\beta$ phase transformation really occurs during the annealing process.

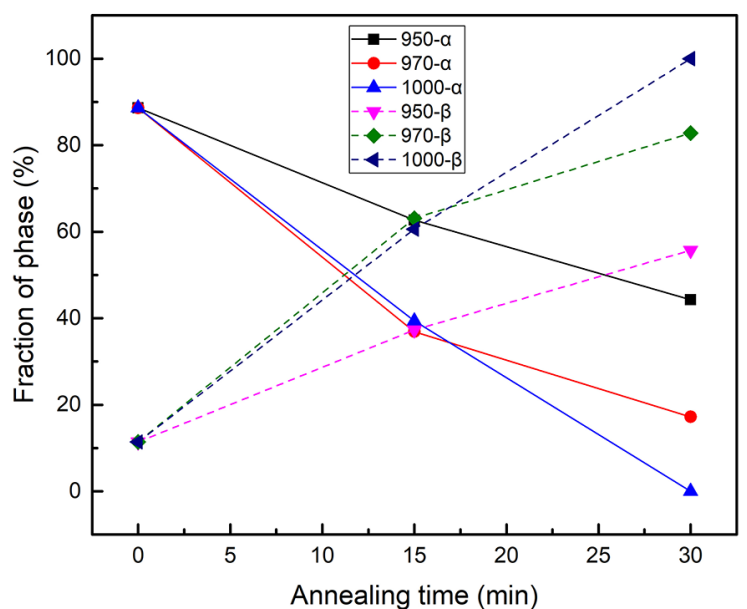

Figure 5. The fractions of $\alpha$ and $\beta$ phases as a function of annealing time at different annealing temperatures.
Meanwhile, the fraction of $\beta$ phase also increases and the fraction of $\alpha$ phase decreases with increasing the annealing temperature.

\section{Discussion}

\subsection{Microstructural evolution and hardness}

Figure 6 shows a schematic diagram of the microstructural evolution of the alloy during isothermal annealing. Red dotted lines in Figure 6a represent initial $\alpha$ grain/subgrain boundaries in the original sample. The green arrows represent the growth direction of $\alpha$ grains. When annealing at high temperature, small-sized $\alpha$ grains will grow and coarsen, with the driving force being the minimization of the interfacial energy ${ }^{21}$. Hence, these small-sized $\alpha$ grains may merge into large grains and the initial grain boundaries will thus disappear. The merged grains will continue to coarsen. When two of these merged $\alpha$ grains grow to meet each other, a "peanut" shape of $\alpha$ grains forms, as seen in Figure $6 \mathrm{~b}$. The $\alpha / \alpha$ grain boundaries (red dotted lines in Figure $6 b$ ) exist among these contiguous grains with "peanut" shape, when an unstable dihedral angle is created at each of the triple point junctions ${ }^{22}$. To minimize surface tension, the dihedral angle will shrink and thus be stabilized by the formation of a groove. The dihedral angle $2 \theta$ is given $b^{23}$ :

$$
2 \cos \theta=\gamma_{a / \beta} / \gamma_{a / a}
$$

where $\gamma_{\alpha / \beta}$ and $\gamma_{\alpha / \alpha}$ represent the $\alpha / \beta$ interface energy and the $\alpha / \alpha$ grain boundary energy, respectively. $\gamma_{\alpha / \beta}$ is a constant and equals to about $0.4 \mathrm{~J} / \mathrm{m}^{22}$. According to Eq. (1), the decrease of $\gamma_{\alpha / \alpha}$ leads to the decrease of the dihedral angle $2 \theta\left(\theta<90^{\circ}\right)$. The decreased dihedral angle means the increased curvature of $\alpha / \beta$ interface. As the high-temperature annealing proceeds, chemical potential gradient occurs between two $\alpha / \beta$ interfaces with different curvatures, which leads to mass transport and thus upsets the local equilibrium of the grooves ${ }^{23}$. Hence, the triple points will move toward each other along the $\alpha / \alpha$ boundaries and meet at last. The elimination of the $\alpha / \alpha$ boundaries means the completion of boundary splitting. Finally, one $\alpha$ grain of "peanut" shape is separated into two
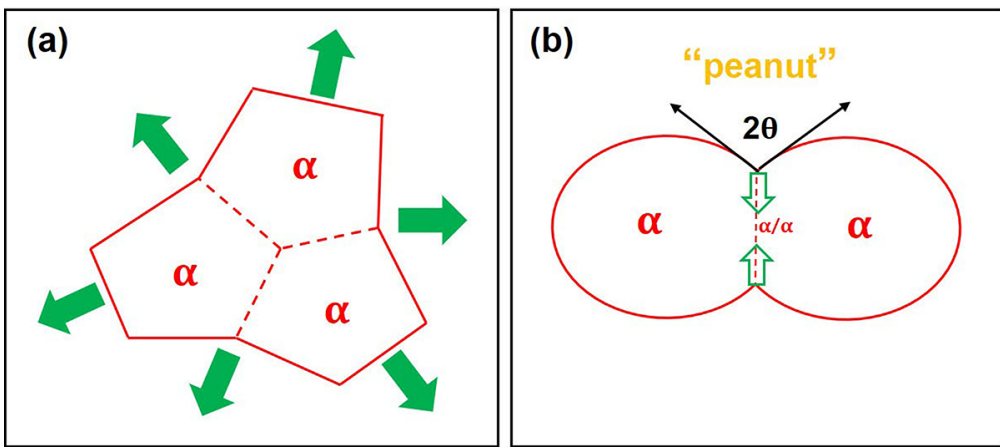

(c)
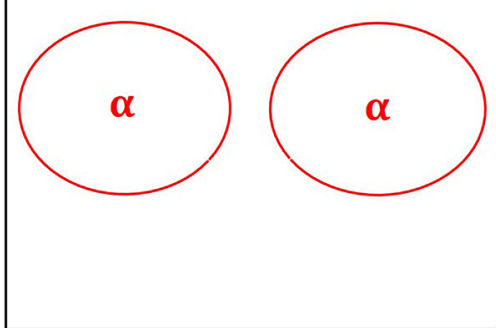

Figure 6. The Schematic diagram of: (a) growth by merging, (b) boundary splitting and (c) the completion of boundary splitting. 
independent equiaxial $\alpha$ grains, as shown in Figure $6 \mathrm{c}$. Furthermore, the $\alpha / \alpha$ boundary can also act as the nucleation site of $\beta$ phase at temperatures around the $\beta$-transus point ${ }^{22}$, as is observed and marked by the small blue ellipse in Figure 4e.

In summary, grains in the $\alpha$ phase first merge into a coarse one in a "peanut" shape, which then splits into two individual single grains below the $\beta$-transus temperature. At temperatures above the $\beta$-transus temperature, however, the $\alpha$ phase disappears absolutely. In comparison, the $\beta$ phase grows continuously at all studied temperatures.

From Figure $4 \mathrm{a}$ to $4 \mathrm{c}$, it can be found the acicular $\alpha^{\prime}$ phase forms in $\beta$ matrix in the involved alloys. Specifically, a large amount of $\alpha$ ' phase is observed in 1000-30 sample. The precipitation of acicular $\alpha^{\prime}$ phases is caused by fast cooling from the high annealing temperature to room temperature. By combining the results of Figure 1 and Figure 4, the increased hardness of all samples is a comprehensive result of grain coarsening and precipitation of $\alpha^{\prime}$ phase. On one hand, grain coarsening in the solution process increases the grain size of alloys, which will lead to a decline in strength and hardness of materials according to the Hall-Petch formula ${ }^{25}$. On the other hand, the acicular $\alpha$ ' phase plays an important role in improving the hardness of alloys. The reason is that the acicular $\alpha^{\prime}$ phase forming inside the $\beta$ matrix can prevent dislocation motion during the plastic deformation. The raise of hardness due to precipitations of $\alpha^{\prime}$ phases surpasses the decrease of hardness caused by grains coarsening. Thus, the hardness of all the specimens increases, compared to that of the original sample.

\subsection{The influence of the diffusion of niobium on the phase transformation}

As mentioned in section 3.3, the $\alpha$-to- $\beta$ phase transformation is accompanied by the diffusion of $\mathrm{Nb}$ at a high solution temperature. According to previous studies ${ }^{10,16}$, pure $\mathrm{Nb}$ has a body-centered cubic structure at arbitrary temperature and has been regarded as a $\beta$-stable element in titanium alloy. Therefore, the diffusion of niobium from $\beta$ to $\alpha$ phase can benefit the phase transformation from $\alpha$ to $\beta$ phase. Figure 7 shows the SEM images of samples 950-15, 950-30 and 1000-30, and the corresponding EDS results with a linear composition analysis of $\beta$ phase. It is found that the $\mathrm{Nb}$ content in grain boundary of $\beta$ phase is higher than that in the interior of phase, when the alloy was annealed below the $\beta$-transus temperature. It confirms that $\mathrm{Nb}$ element diffuses from $\beta$ to $\alpha$ phase
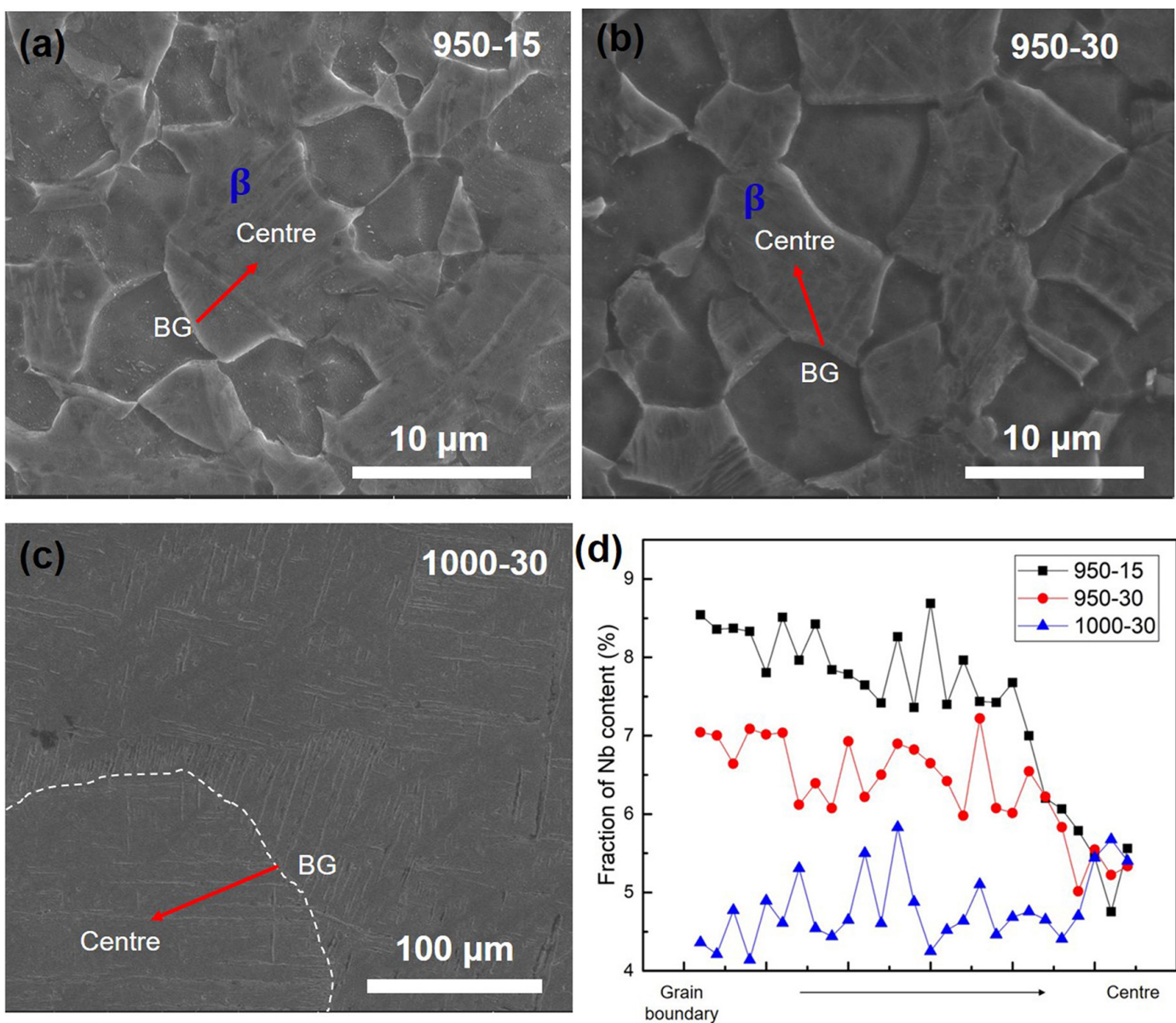

Figure 7. The regions of line scanning of (a) sample 950-15, (b) sample 950-30 and sample 1000-30, and (d) the linear composition analysis of $\mathrm{Nb}$ content of in samples 950-15, 950-30 and 1000-30. 
during the annealing process. It is also found that the $\mathrm{Nb}$ content of $\beta$ phase from grain boundary to the grain interior is basically unchanged, when the alloy was annealed above the $\beta$-transus temperature. It shows that $\alpha$ phase has been completely transformed into $\beta$ phase. Figure 8 a shows a schematic diagram of the $\mathrm{Nb}$ diffusion from $\beta$ to $\alpha$ phase and its effect on the $\alpha$-to- $\beta$ phase transformation. In Figure $8 \mathrm{a}$, red grain represents $\alpha$ phase, blue grains represent $\beta$ phase, and navy-blue circles and green arrows represent niobium atoms and migration direction of $\alpha$ grain boundary, respectively.

The impact of $\mathrm{Nb}$ diffusion on the $\alpha$-to- $\beta$ phase transformation is investigated by DFT calculations, where thermodynamic stability of HCP and BCC titanium with different niobium contents is discussed. The formation energy is a physical quantity that determines thermodynamic stability of materials. The formation energy per atom $E_{f}\left(T i_{x} N b_{1-x}\right)$, of Ti-Nb alloy is defined as:

$$
\begin{gathered}
E_{f}\left(T i_{x} N b_{1-x}\right)=E_{t o t}\left(T i_{x} N b_{1-x}\right) / N-x \\
\mu^{T i}(H C P-b u l k)-(1-x) \cdot \mu^{N b}(B C C-b u l k)
\end{gathered}
$$

(a)

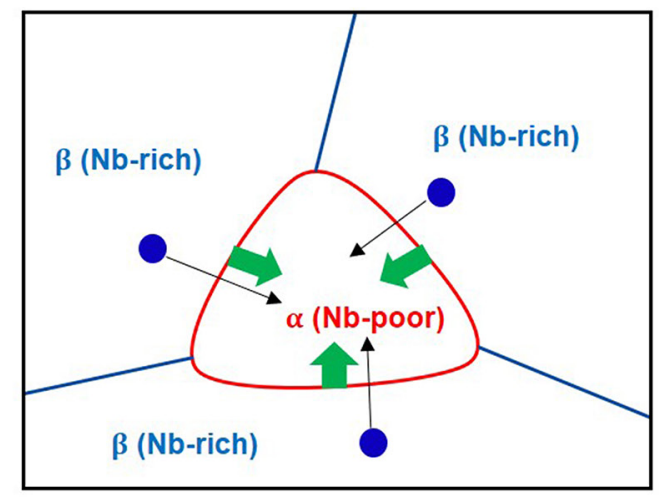

(c)

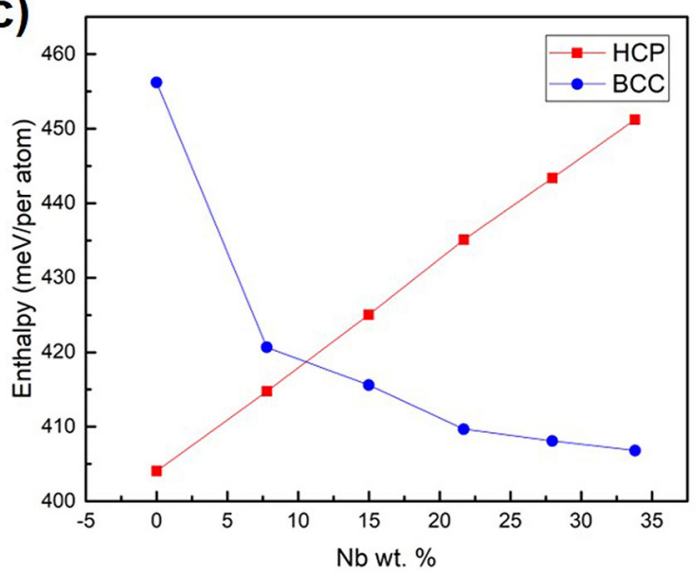

where $N$ is the total number of atoms in the supercell (HCP), $x$ is the compositional fraction of Ti, $\mathrm{E}_{\text {tot }}$ is the total energy of the supercell (HCP), $\mu^{T i}$ and $\mu^{N b}$ are the chemical potentials of bulk $\mathrm{Ti}$ and $\mathrm{Nb}$, respectively. When $E_{f}<0$, the alloy is thermodynamically stable.

In order to calculate the formation energy of Ti-Nb alloy at elevated temperature, the enthalpy of Ti-Nb alloy is firstly calculated and defined $\mathrm{as}^{26}$ :

$$
\Delta H_{T}\left(T i_{x} N b_{1-x}\right)=\Delta H_{0}\left(T i_{x} N b_{1-x}\right)+\int_{0}^{T} \bar{C}_{p}^{T i-N b} d T
$$

where $\Delta H_{T}\left(T i_{x} N b_{1-x}\right)$ is the enthalpy of Ti-Nb alloy at $T \mathrm{~K}$, $\Delta H_{0}\left(T i_{x} N b_{1-x}\right)$ is the enthalpy of Ti-Nb alloy at $0 \mathrm{~K}, \bar{C}_{p}^{H-N b}$ is the heat capacity of Ti-Nb alloy and is given by ${ }^{27}$ :

$$
\bar{C}_{p}^{T i-N b}=x \cdot c_{p}^{T i}+(1-x) \cdot C_{p}^{N b}
$$

where $C_{p}^{T i}$ and $C_{p}^{N b}$ are the heat capacity of pure titanium and pure niobium, respectively ${ }^{28}$. It should be noted that the volume change is neglected due to its small effect on enthalpy. Therefore, the enthalpy of Ti-Nb alloy at $0 \mathrm{~K}, \Delta \mathrm{H}_{0}$ $\left(T i_{x} N b_{1-x}\right)$, is equal to the formation energy $\left(E_{f}\left(T i_{x} N b_{1-x}\right)\right)$ at $0 \mathrm{~K}$.

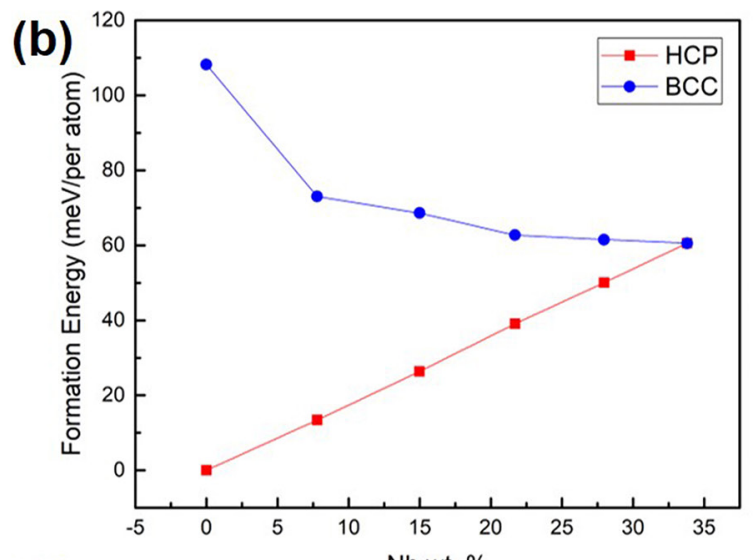

(d)

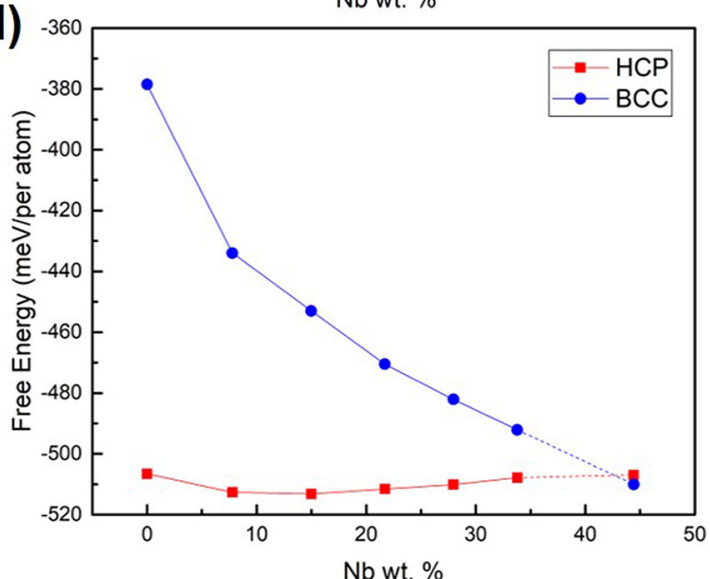

Figure 8. The Schematic diagram of: (a) the diffusion of niobium from BCC to HCP phase, (b) the phase transformation from HCP to BCC phase after the diffusion, (c) the relationship between $\mathrm{Nb}$ concentration and formation energy of $\mathrm{HCP}$ and $\mathrm{BCC}$ phase at $0 \mathrm{~K}$, (d) the relationship between $\mathrm{Nb}$ concentration and the enthalpy of $\mathrm{HCP}$ and $\mathrm{BCC}$ phase at $1155 \mathrm{~K}$, and (e) the relationship between $\mathrm{Nb}$ concentration and free energy of $\mathrm{HCP}$ and $\mathrm{BCC}$ phase at $1155 \mathrm{~K}$. 
Furthermore, the entropy is also considered. The entropy for alloys at all temperatures consists of two parts: the mixing entropy and vibration entropy ${ }^{29}$. The ideal mixing entropy depends on the composition of alloys but not on local atomic arrangements. The ideal mixing entropy and vibration entropy are respectively given by ${ }^{10,26 \text { : }}$

$$
\begin{gathered}
S_{\text {mixing }}(x)=k \cdot[x \cdot \ln (x)+(1-x) \cdot \ln (1-x)] \\
S_{\text {ribration }}(x)=x \cdot\left(S_{298}^{T i}+\int_{298}^{T} \frac{C_{p}^{T i}}{T} d T\right) \\
+(1-x) \cdot\left(S_{298}^{N b}+\int_{298}^{T} \frac{C_{p}^{N b}}{T} d T\right)
\end{gathered}
$$

where $k$ is the Boltzmann constant, $S_{298}^{T_{i}}$ and $S_{298}^{N b}$ are the entropy of pure titanium and pure niobium at $298 \mathrm{~K}$, respectively ${ }^{28}$. The temperature dependence of the free energy can be calculated by ${ }^{26}$ :

$$
\begin{gathered}
\Delta F_{f}(x, T)=\Delta H_{T}\left(T i_{x} N b_{1-x}\right)- \\
T \cdot\left(S_{\text {mixing }}(x)+S_{\text {vibration }}(x)\right)
\end{gathered}
$$

Based on Eqs. (2) to (7), the enthalpies, $\Delta H_{T}\left(T i_{x} N b_{1-x}\right)$ of the $T i_{x} N b_{1-x}$ alloys in the HCP and BCC structures as a function of the $\mathrm{Nb}$ contents are indicated in Figure 8c, while the corresponding free energies, $\Delta F_{f}(x, T)$, of the $T i_{x} N b_{1-x}$ alloys are shown in Figure 8d. Those enthalpies and free energies are calculated at $0 \mathrm{~K}$ and $1155 \mathrm{~K}$, respectively. The latter temperature $(1155 \mathrm{~K})$ represents the $\mathrm{HCP}-\mathrm{BCC}$ transition temperature in pure Ti when the HCP and BCC phases can coexist in the material simultaneously. Therefore, the temperature of 1155 $\mathrm{K}$ is adopted when calculating the energies of the $T i_{x} N b_{1-x}$ alloys by DFT calculations in the present study.

From Figure 8b, 8c and 8d, the energy (the enthalpy and free energy) of the $T i_{x} N b_{1-x}$ alloys in the HCP structure increases while that in the BCC structure decreases with increasing the $\mathrm{Nb}$ content. When the $\mathrm{Nb}$ content reaches a specific critical value, the energy of HCP equals that of BCC, indicating an equilibrium state between the $\mathrm{HCP}$ and $\mathrm{BCC}$ phases. Below that critical value of $\mathrm{Nb}$ content, the $\mathrm{HCP}$ phase represents the stable phase, compared to the BCC phase. However, for the alloys with $\mathrm{Nb}$ content above the critical value, the energy of HCP is greater than that of BCC, thus the HCP phase tends to transform to the stable BCC phase. Therefore, when the average $\mathrm{Nb}$ content in the alloy exceeds the specific critical value, the BCC phase acts as the stable phase, compared to the HCP phase. Specifically, for $T=0 \mathrm{~K}$, the critical content of $\mathrm{Nb}$ element is about $33 \%$, as shown in Figure $8 \mathrm{~b}$. As the temperature increases up to $1155 \mathrm{~K}$, the critical value of the $\mathrm{Nb}$ element decreases to about $11 \%$ provided that only the enthalpy but not the entropy is considered, as indicated by Figure 8c. However, the results in Figure 8d show that the mixing and vibration entropies plays a significant role in determining the equilibrium state between the HCP and $\mathrm{BCC}$ phases at an elevated temperature of $1155 \mathrm{~K}$.
Predicting by drawing extension lines, the free energy of those two phases equals when the content of $\mathrm{Nb}$ element reaches about $43 \%$.

By combining the experimental results in the present study, as shown by Figure $3 \mathrm{a}, 3 \mathrm{c}$ and $3 \mathrm{~d}$, the alloying $\mathrm{Nb}$ element is enriched in the BCC phase but poor in the HCP phase. The concentration gradient thus leads to the diffusion of $\mathrm{Nb}$ from the BCC phase to the HCP phase. Considering the small volume fraction of the HCP phase compared to that of the BCC phase in the annealed titanium alloys, such diffusion has minor effect on the concentration of $\mathrm{Nb}$ element in the BCC phase but may greatly promote the corresponding content of $\mathrm{Nb}$ in the HCP phase. According to the DFT calculations, as shown in Figure 8b, 8c and 8d, the stability of the HCP phase declines as the concentration of $\mathrm{Nb}$ increases in the $\mathrm{HCP}$ phase. Moreover, when the $\mathrm{Nb}$ content in the HCP exceeds a specific value, the HCP phase transforms to the BCC phase. The first principle DFT calculations illustrates that the diffusion of $\mathrm{Nb}$ from the $\mathrm{BCC}$ to $\mathrm{HCP}$ phase will promote the $\mathrm{HCP}$-to-BCC transformation in the Ti-Nb alloys during heat treatment, which conforms to the observations by experiment in the present study. Nonetheless, it should be noted that the theoretical models by DFT calculations provide a relatively high critical value of the $\mathrm{Nb}$ content about $43 \%$ for the equilibrium state between the HCP and BCC phases. Although it is difficult for the present study to detect the exact concentration of $\mathrm{Nb}$ distributing in the grains due to their small sizes, it is expected that discrepancies may exist between the theoretical value and experimental results. The possible reasons are included as follows. Firstly, both the calculation method and theoretical models involve approximation processing, for example, the enthalpies and free energies at elevated temperatures are estimated according to empirical or theoretical expressions. Secondly, the alloying element aluminum is exempted from the Ti-Nb alloys during the DFT calculations. Thirdly, the annealing temperature in the experiments are about $100 \mathrm{~K}$ higher than the adopted temperature $(1155 \mathrm{~K})$ in calculations. In spite of that, the DFT calculations in the present study provide reliable and reasonable clues in illustrating the experimental observations. That is, the diffusion of niobium from $\beta$ to $\alpha$ phase benefits the phase transformation from $\alpha$ to $\beta$ phase in titanium alloys.

\subsection{Martensitic transformation}

Figure 9 shows the EBSD micrographs and pole figures for $\beta$ and $\alpha^{\prime}$ phases of 1000-30 sample. It can be seen from Figure 9a that the $\alpha^{\prime}$ and $\beta$ phases orient in the Burgers orientation relationship of $\{110\}_{\text {bcc }}\left\|\{0001\}_{\text {hcp }},<111>_{\text {bcc }}\right\|<11 \overline{2} 0>_{\text {hcp }}{ }^{30}$.

According to the ab initio calculation by EKMAN et $a l .{ }^{31}$, the BCC structure transforms to the HCP structure via two steps, as indicated in Figure 10. In the first step, shear movement of atoms takes place on every second $\{110\}_{\text {bcc }}$ planes (solid red circles) along the $<111>_{\text {bcc }}$ directions, during which the involving atoms move from the original position to the saddle point (dotted red circles). 


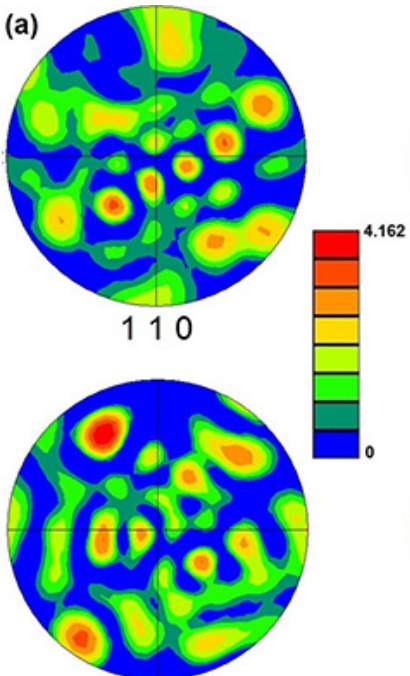

111

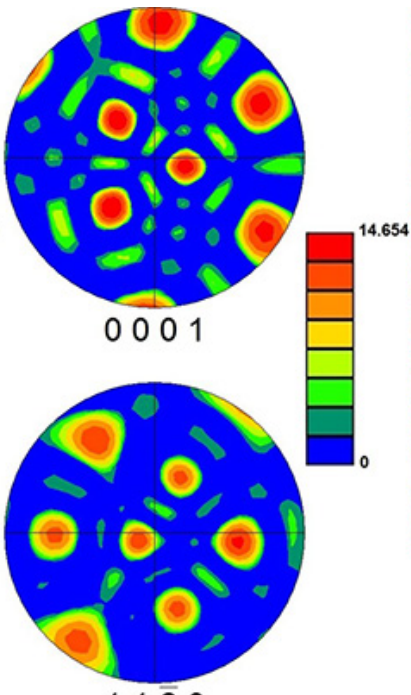

$1 \overline{1} \overline{2} 0$

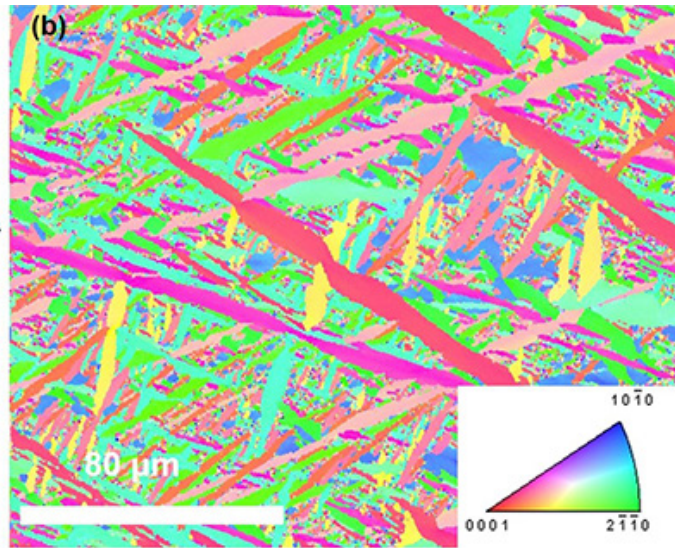

Figure 9. (a) The pole figures for $\beta$ and $\alpha^{\prime}$ phases and (b) EBSD image of 1000-30 sample.
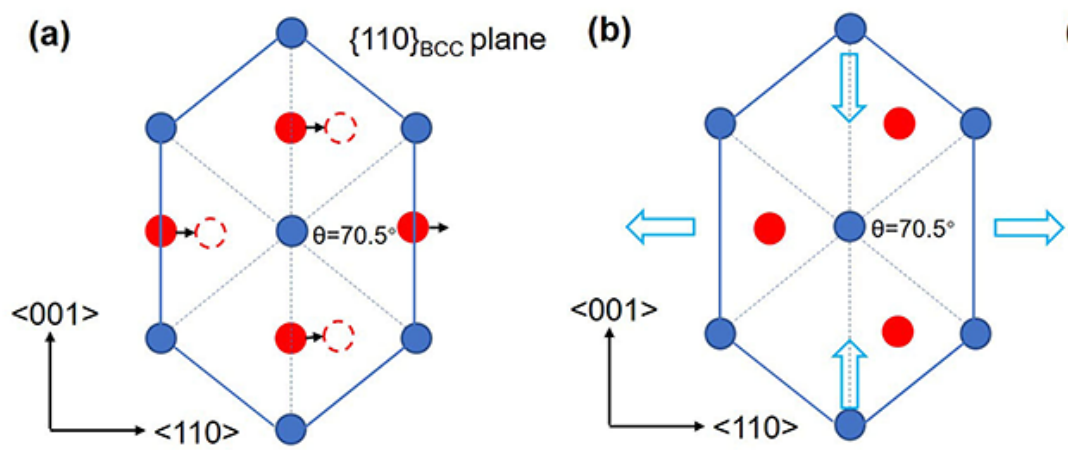

(c)

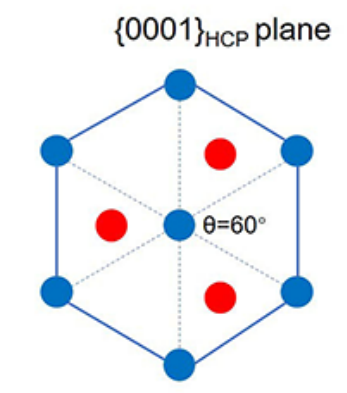

Figure 10. The schematic diagram of martensitic transformation mechanism from BCC to HCP structure.

In the second step, the original BCC lattice shrinks in the $<001\rangle_{\text {bcc }}$ direction and dilates along the $\langle 110\rangle_{\text {bcc }}$ direction simultaneously on the $\{110\}_{\beta}$ planes. After the two steps, the BCC structure transforms absolutely into the HCP structure, during which the value of $\theta$ decreases from $70.5^{\circ}$ to $60^{\circ}$ and the volume of the unit cell increases as well.

\section{Conclusions}

This paper studies the microstructural evolution and hardness of a Ti-5Nb-5Al alloy under different annealing treatment conditions. The results show that grains first merge into a coarse one in a "peanut" shape, which then splits into two individual single grains below the $\beta$-transus temperature, but disappears absolutely above the $\beta$-transus temperature. Compared to $\alpha$ phase, the $\beta$ phase shows continuous growth at all the studied temperatures. The slight raise of hardness of the alloy after heat treatment is a combined result of grain coarsening and precipitation of $\alpha^{\prime}$ phase. The effect of the niobium diffusion on the $\alpha$-to- $\beta$ transformation in the titanium alloy was also investigated by first principle calculations.
According to the first-principle DFT calculations, the diffusion of niobium from $\beta$ to $\alpha$ phase promoted the phase transformation from $\alpha$ to $\beta$ phase.

\section{Acknowledgments}

This work is financially supported by National Natural Science Foundation of China (grant number 51828102).

\section{References}

1. Warchomicka F, Poletti CM, Stockinger M. Study of the hot deformation behavior in Ti-5Al-5Mo-5V-3Cr-1Zr. Materials Science and Engineering: A. 2011;528:8277-8285.\{, \#1\}

2. Warchomicka F, Stockinger M, Degischer HP. Quantitative analysis of the microstructure of near $\beta$ titanium alloy during compression tests. Journal of Materials Processing Technology. 2006;177(1-3):473-477.

3. Wang X, Jahazi M, Yue S. Substructure of high temperature compressed titanium alloy IMI 834. Materials Science and Engineering: A. 2006;434(1-2):188-193. 
4. Picu RC, Majorell A. Mechanical behavior of Ti-6Al-4V at high and moderate Temperatures-Part II: constitutive modeling. Materials Science and Engineering: A. 2002;326:306-316.

5. Zhang ZX, Qu SJ, Feng AH, Shen J, Chen DL. Hot deformation behavior of Ti-6Al-4V alloy: Effect of initial microstructure. Journal of Alloys and Compounds. 2017;718:170-181.

6. Huang CW, Zhao YQ, Xin SW, Zhou W, Li Q, Zeng WD. Effect of microstructure on tensile properties of Ti-5Al-5Mo-5V-3Cr- $1 \mathrm{Zr}$ alloy. Journal of Alloys and Compounds. 2017;693:582-591.

7. Shi ZF, Guo HZ, Han JY, Yao ZK. Microstructure and mechanical properties of TC21 titanium alloy after heat treatment. Transactions of Nonferrous Metals Society of China. 2013;23:2882-2889.

8. Zhao ZB, Wang QJ, Hu QM, Liu JR, Yu BB, Yang R. Effect of $\beta$ (110) texture intensity on $\alpha$-variant selection and microstructure morphology during $\beta / \alpha$ phase transformation in near $\alpha$ titanium alloy. Acta Materialia. 2017;126:372-382.

9. Dong RF, Li J, Fan J, Kou HC, Tang B. Precipitation of $\alpha$ phase and its morphological evolution during continuous heating in a near $\beta$ titanium alloy Ti-7333. Materials Characterization. 2017;132:199-204.

10. Raabe D, Sander B, Friák M, Ma D, Neugebauer J. Theoryguided bottom-up design of $\beta$-titanium alloys as biomaterials based on first principles calculations: Theory and experiments. Acta Materialia. 2007;55(13):4475-4487.

11. Li C, Zhang XY, Zhou KC, Peng CQ. Relationship between lamellar $\alpha$ evolution and flow behavior during isothermal deformation of Ti-5 Al-5Mo-5V-1Cr-1Fe near $\beta$ titanium alloy. Materials Science and Engineering: A. 2012;558:668-674.

12. Li T, Kent D, Sha G, Liu HW, Friesg SG, Ceguerra AV, et al. Nucleation driving force for $\omega$-assisted formation of $\alpha$ and associated $\omega$ morphology in $\beta$-Ti alloys. Scripta Materialia. 2018;155:149-154.

13. Sadeghpour S, Abbasi SM, Morakabati M, Karjalainen LP, Porter DA. Effect of cold rolling and subsequent annealing on grain refinement of a beta titanium alloy showing stress-induced martensitic transformation. Materials Science and Engineering: A. 2018;731:465-478.

14. Kharia KK, Rack HJ. Martensitic phase transformations in IMI 550 (Ti-4Al-4Mo-2Sn-0.5Si). Metallurgical and Materials Transactions: A. 2001;32(3):671-679.

15. Minshin Y, Herzig C. Diffusion in the Ti-Al system. Acta Materialia. 2000;48(3):589-623.

16. Niinomi M. Mechanical properties of biomedical titanium alloys. Materials Science and Engineering: A. 1998;243(1-2):231-236.
17. Kresse G, Hafner J. Ab initio molecular dynamics for open-shell transition metals. Physical Review: B. 1993;48(17):13115-13118.

18. Kresse G, Furthmut J. Efficient iterative schemes for ab initio total-energy calculations using a plane-wave basis set. Physical Review: B. 1996;54(16):15-50

19. Perdew JP, Burke K, Ernzerhof M. Generalized gradient approximation made simple. Physical Review Letters. 1996;77(18):3865-3868.

20. Kresse G, Joubert D. From ultrasoft pseudopotentials to the projector augmented-wave method. Physical Review B. 1999;59(3):1758-1775.

21. Martin JW, Doherty RD. Cantor B. Stability of Microstructure in Metallic Systems. $2^{\text {nd }}$ ed. Cambridge: Cambridge University Press; 1997.

22. Gao XX, Zeng WD, Wang YB, Long Y, Zhang SF, Wang QJ. Evolution of equiaxed alpha phase during heat treatment in a near alpha titanium alloy. Journal of Alloys and Compounds. 2017;725:536-543.

23. Sharma G, Ramanujan RV, Tiwari GP. Instability mechanisms in lamellar microstructures. Acta Materialia. 2000;48(4):875-889.

24. Park CH, Won JW, Park JW, Semiatin SL, Lee CS. Mechanisms and kinetics of static spheroidization of hot-worked Ti-6Al-2Sn4Zr-2Mo-0.1Si with a lamellar microstructure. Metallurgical and Materials Transactions: A. 2012;43(3):977-985.

25. Yu HH, Xin YC, Wang MY, Liu Q. Hall-Petch relationship in Mg alloys: A review. Journal of Materials Science and Technology. 2018;34(2):248-256.

26. Saunders N, Miodownik AP. CALPHAD (calculation of phase diagrams): a comprehensive guide. $1^{\text {st }}$ ed. New York: Elsevier; 1998.

27. Zienert T, Leineweber A, Fabrichnaya O. Heat capacity of Fe-Al intermetallics: B2-FeAl, $\mathrm{FeAl}_{2}, \mathrm{Fe}_{2} \mathrm{Al}_{5}$ and $\mathrm{Fe}_{4} \mathrm{Al}_{13}$. Journal of Alloys and Compounds. 2017;725:848-859.

28. Brandes EA, Brook GB. Smithells Metals Reference Book. $7^{\text {th }}$ ed. Oxford: Educational and Professional Publishing; 1998.

29. Turchi PEA, Drchal V, Kudrnovsky J, Colinet C, Kaufman L, Liu ZK. Application of ab initio and CALPHAD thermodynamics to Mo-Ta-W alloys. Physical Review: B. 2005;71(9):094206-094219.

30. Burgers WG. On the process of transition of the cubic-body-centered modification into the hexagonal-close-packed modification of zirconium. Physica. 1934;1(7-12):561-586.

31. Ekman M, Sadigh B, Einarsdotter K, Blaha P. Ab initio study of the martensitic bcc-hcp transformation in iron. Physical Review: B. 1998;58(9):5296-5305. 